\title{
Controversies in the Management of Early-Stage Hodgkin Lymphoma
}

\author{
Presented by Ranjana H. Advani, MD
}

\begin{abstract}
Early-stage Hodgkin lymphoma is a highly curable malignancy, but controversies surrounding treatment recommendations persist due to the sheer number of treatment choices available, as well as the effort to balance risk versus benefit for each individual patient. The gold standard for treatment has evolved over the years. Currently, in the PET era, fine-tuning therapy approaches is largely focused on avoiding giving too much therapy to patients with a negative interim PET and too little therapy to those with a positive interim PET. Careful patient selection for therapy has become increasingly important, as patient risk factors for early-stage disease are variably defined by German Hodgkin Study Group, EORTC, and NCCN criteria.
\end{abstract}

Common controversies in the management of earlystage Hodgkin lymphoma (HL) boil down to balancing the risk/benefit profile of treatment for each individual, according to Ranjana H. Advani, MD, Professor of Lymphoma, Stanford Cancer Institute. "We need to have the highest cure rate with primary therapy, but we also have to remember to optimize survivorship," she said at the NCCN 2020 Virtual Congress: Hematologic Malignancies.

The gold standard of treatment for stage I-II classic HL has evolved significantly over the past 50 years, from extensive radiation therapy (RT) in the late 1960s to 1970 s, to aggressive combined modality therapy (CMT) with extended-field RT from the late 1970s to early 1990s, to reduced-intensity CMT with involved-field RT (IFRT) in the mid-1990s, to an even further reduction in chemotherapy with involved-node/involved-site RT (INRT/ISRT) in the mid-2000s.

"Now, we're using PET-adapted strategies to assess if RT can be totally eliminated, and with 3 novel agents available and approved for relapsed disease, we're seeing how those can be incorporated into frontline therapy," said Dr. Advani. These gold standards evolved over time because of the recognition of late sequelae in HL survivors (eg, increased risk of second cancers and cardiovascular disease), largely from the era in which extended-field RT (EFRT) was used. ${ }^{1}$

"Over time, exposure of normal breast tissue or the heart [to radiation] has decreased substantially. Current data show that survival from HL is now tracking along with second cancers and cardiac events, so it looks like the evolution of RT has made a difference," she said; however, longer follow-up is required. ${ }^{2}$

\section{Defining the Disease Burden}

According to Dr. Advani, defining a patient's disease burden to assess prognosis is critical in early-stage disease and requires an understanding of the heterogeneity of early-stage HL. "To understand prognosis is to understand how risk is defined," she said. This risk varies based criteria from the German Hodgkin Study Group (GHSG), EORTC, or NCCN (Figure 1).

"It's important to understand these differences, because as we apply results of trials, we have to understand the characteristics of patients enrolled on these trials, so that we can then apply the results to the appropriate patient population," she said.

\section{Current Treatment Approaches}

CMT consisting of 4 to 6 cycles of chemotherapy with ABVD (doxorubicin/bleomycin/vinblastine/dacarbazine) + IFRT (30 Gy) has been an established standard of care for early-stage HL for decades, with response determined by traditional CT imaging.

According to Dr. Advani, goals of therapy in 2020 are dictated by finding the right balance between efficacy and long-term toxicity. More recent studies in the CT era had attempted to fine-tune CMT, with the goal of avoiding giving too much therapy for patients with favorable disease and too little for those with unfavorable disease. Now, in the PET era, understanding the Deauville 5-point scoring system is critical. "It's important that you educate your nuclear medicine physicians on reporting scans using this scoring system because that makes a big difference in how you are going to determine therapy," she said. "This is a visual score, and 
UNFAVORABLE RISK FACTORS FOR STAGE I-II CLASSIC HODGKIN LYMPHOMA

\begin{tabular}{|l|l|l|l|}
\hline Risk Factor & GHSG & EORTC & NCCN \\
\hline Age & & $\geq 50$ & \\
\hline Histology & & & \\
\hline ESR and B symptoms & $>50$ if $\mathrm{A}_{;}>30$ if $\mathrm{B}$ & $>50$ if $\mathrm{A}_{i}>30$ if B & $\geq 50$ or any $\mathrm{B}$ symptoms \\
\hline Mediastinal mass & MMR $>0.33$ & MTR $>0.35$ & MMR $>0.33$ \\
\hline \# Nodal sites & $>2^{*}$ & $>3^{*}$ & $>3$ \\
\hline E lesion & any & & \\
\hline Bulky & & & $>10 \mathrm{~cm}$ \\
\hline
\end{tabular}

Figure 1. Risk definitions in early-stage Hodgkin lymphoma: GHSG, EORTC, and NCCN.

Abbreviations: GHSG, German Hodgkin Study Group; MMR, mediastinal mass ratio, maximum width of mass/maximum intrathoracic diameter; MTR, mediastinal thoracic ratio, maximum width of mediastinal mass/maximum diameter at T5-6.

From the NCCN Clinical Practice Guidelines in Oncology: Hodgkin Lymphoma. Version 2.2020. @ 2020 National Comprehensive Cancer Network, All rights reserved. This illustration may not be reproduced in any form without the express written permission of NCCN.

it is really up to the nuclear medicine physicians to provide this to clinicians."

\section{Can We Distinguish "Good" From "Bad" Disease?}

The question arises as to whether therapy modification based on interim PET scan has the potential to select patients for treatment escalation or de-escalation. For these modifications to potentially improve outcomes, the negative predictive value should be very high (ie, most patients could be cured with continuing or de-escalating primary therapy) and PET-positive patients should be salvageable with alternative therapy.

"Keeping to the theme of avoiding giving too much therapy to patients with a negative interim PET scan, and too little to those with a positive interim PET, the important thing is remembering the criteria used to define a negative interim PET and a positive interim PET," she said. "The Europeans (GHSG and EORTC) use a more conservative definition of a negative PET." A score of 1 to 2 to define a negative PET, whereas the CALGB/Alliance 50604 Group uses a score of 1 to 3. Conversely, to define a positive PET, the Europeans use a score of 3 to 5 and CALGB/Alliance 50604 uses a score of 4 to 5 .

\section{Avoiding Too Much Therapy}

Final results of the randomized phase III HD16 trial by the GHSG in a very favorable group of patients demonstrated that $20 \mathrm{~Gy}$ RT is still required if a patient is PET-negative after 2 cycles of ABVD. ${ }^{3}$ "So you can't get away with just 2 cycles of ABVD. Even for the most favorable patients, you drop from a 5-year progression-free survival [PFS] rate of $93 \%$ to $86 \%$, and for a curable disease, that's unacceptable," she said. "It is clear that if you're trying to get rid of RT, you have to increase the number of cycles of chemotherapy."

The GHSG HD16 study also showed that despite RT, patients with a Deauville score of 4 to 5 had inferior PFS compared with those with a Deauville score of 1 to 3 . "So, for patients with a Deauville score of 4 to 5 after 2 cycles of ABVD, it's probably not wise to radiate at that point, and one should continue with 2 additional cycles of chemotherapy," she said.

The EORTC H10 trial revealed similar findings in favorable patients who were PET-negative and received RT after 3 cycles of ABVD, with a 5-year PFS benefit of $99 \%$ versus $87.1 \%$ in patients treated with 3 cycles of ABVD alone. ${ }^{4}$ In unfavorable patients the magnitude of benefit with RT was only $2.5 \%$ because 6 cycles of chemotherapy was used (rather than 4) among patients who did not receive RT.

A UK trial showed similar results in patients who were PET-negative. ${ }^{5}$ Patients who received RT after 3 cycles of ABVD had better outcomes than those who received no further treatment, with no difference in overall survival (because patients had early-stage disease). In patients with unfavorable early-stage disease, results from the GHSG HD17 trial suggested that RT can be omitted if a patient is PET-negative (Deauville score is $1-2$ ) after $2 \times 2$ [2x escalated BEACOPP $+2 \mathrm{x}$ ABVD], Dr. Advani said (ClinicalTrials.gov identifier: NCT01356680).

Based on these collective study findings, Dr. Advani concluded that consolidative RT continues to be controversial in patients with interim PET-negative disease but is appropriate for those with a positive interim or end-of-therapy PET scan. INRT/ISRT appears adequate to prevent relapse, with likely fewer long-term/late effects than previously seen with EFRT. "And it's really important to have long-term follow-up because these late effects don't happen in the short term," she noted. "You need 20 or 30 years of follow-up."

\section{Applying the Data to Day-to-Day Practice}

According to Dr. Advani, certain factors should be considered to help individualize therapy and assess a patient's risk from primary therapy: the patient's age and sex, the anatomic extent of their disease and resultant normal tissue exposure to RT, the cumulative toxicity of additional cycles of chemotherapy if RT is 
avoided, and any added toxicity from salvage therapy. "You have to define disease distribution for therapy selection," she said.

Optimizing/individualizing therapy requires a balance between immediate cure and prevention of late toxicity, she added. Considerations for chemotherapy alone should include women aged $<35$ years with axillary and/or mediastinal involvement, where there is inability to limit the RT dose to important cardiac subunits. CMT should be considered in patients with favorable disease, especially when it is possible to limit the duration of chemotherapy; patients with a positive interim PET scan $(\sim 25 \%)$; patients with bulky adenopathy; and in geographic areas where stem cell or PET imaging is not easily available or is cost-prohibitive.

\section{Emerging Treatment Approaches For Early-Stage HL}

According to Dr. Advani, emerging approaches focus mainly on incorporating novel agents in the frontline setting with brentuximab vedotin (BV) and checkpoint inhibitors (nivolumab, pembrolizumab), and on the identification of biological subsets to guide therapy.

Recent research has focused on determining whether the addition of BV might allow for a reduction or even omission in the ISRT dose, with the hope of minimizing acute and late effects of treatment in patients with unfavorable early-stage HL. ${ }^{6}$ She noted that the results of this Memorial Sloan Kettering Cancer Center trial were comparable to those of the HD11 and HD14 studies in similar populations, so although adding BV to the frontline setting is feasible, long-term follow-up is needed and cost considerations remain a concern.

Focusing on checkpoint inhibitors, the randomized phase II NIVAHL trial examined the concurrent administration of nivolumab, AVD, and ISRT versus sequential administration in early-stage unfavorable HL. ${ }^{7}$ "At the end of treatment, the complete response rate was pretty identical in both arms," she said. "There were no increased safety signals after a median follow-up of 14 months, but we need to see longer-term follow-up of this data."

Emerging prognostic markers in HL will focus on reclassifying patients with early-stage HL based on functional radiographic markers (eg, tumor metabolic volume, total lesion glycolysis) at presentation. ${ }^{8}$ "The currently used Deauville score doesn't tell you anything upfront; it tells you something when you're already in the middle of therapy," she said. "“These markers are still not incorporated into reports, but I suspect that's where the field is moving."

Dr. Advani circled back to the importance of optimizing survivorship, and emphasized the importance of setting up survivorship programs for adult lymphoma patients.

"Finally, there is a disturbing association between facility volume and mortality of patients with HL," she said. " "This is one of the most highly curable diseases, with a median age of 30 years at time of diagnosis, but outcomes are best in large-volume centers," she added. "So if you are in a center that sees $<9$ to 10 patients a year, reach out to centers of expertise and look at the NCCN Guidelines, as they have been developed to guide physicians in state-of-the-art practice. Because at the end of the day, the goal is to cure the patient while giving them the best quality of life."

\footnotetext{
Disclosures: Dr. Advani has disclosed that she has received consulting fees from ADC Therapeutics, Celgene Corporation, Genentech, Inc./Roche Laboratories, Inc., Gilead Sciences, Inc., Portola Pharmaceuticals, Inc., Sanofi-Aventis U.S., Seattle Genetics, Inc., Kura Oncology, Inc, Karyopharm, and Takeda Pharmaceuticals North America, Inc.; and received institutional grant/research support from Forty Seven, Inc. Genentech, Inc./Roche Laboratories, Inc., Janssen Pharmaceutica Products, LP, Kura Oncology, Inc., Merck \& Co., Inc., Millennium Pharmaceuticals, Inc., Regeneron Pharmaceuticals, Inc., and Seattle Genetics, Inc.
}

Correspondence: Ranjana H. Advani, MD, Stanford Cancer Institute, 875 Blake Wilbur Drive, Stanford, CA 94305. Email: radvani@stanford.edu

\section{References}

1. van Leeuwen $\mathrm{FE}, \mathrm{Ng} \mathrm{AK}$. Late sequelae in Hodgkin lymphoma survivors Hematol Oncol 2017;35(Suppl 1):60-66.

2. Patel $C G$, Michaelson $E$, Chen $Y$, et al. Reduced mortality risk in the recent era in early-stage Hodgkin lymphoma patients treated with radiation therapy with or without chemotherapy. Int J Radiat Oncol Biol Phys 2018; 100:498-506.

3. Fuchs M, Goergen H, Kobe C, et al. Positron emission tomography-guided treatment in early-stage favorable Hodgkin lymphoma: final results of the international, randomized phase III HD16 trial by the German Hodgkin Study Group. J Clin Oncol 2019;37:2835-2845.

4. André MPE, Girinsky T, Federico M, et al. Early positron emission tomography response-adapted treatment in stage I and II Hodgkin lymphoma: final results of the randomized EORTC/LYSA/FIL H10 trial. J Clin Oncol 2017;35:1786-1794.

5. Radford J, Illidge T, Counsell N, et al. Results of a trial of PET-directed therapy for early-stage Hodgkin's lymphoma. N Engl J Med 2015;372: 1598-1607.

6. Kumar A, Casulo C, Yahalom J et al. Brentuximab vedotin and AVD followed by involved-site radiotherapy in early stage, unfavorable risk Hodgkin lymphoma. Blood 2016;128:1458-1464.

7. Brockelmann PJ, Goergen $\mathrm{H}$, Keller U, et al. Efficacy of nivolumab and AVD in early-stage unfavorable classic Hodgkin lymphoma: the randomized phase 2 German Hodgkin Study Group NIVAHL trial. JAMA Oncol 2020;6:872-880.

8. Cottereau AS, Versari A, Loft A, et al. Prognostic value of baseline metabolic tumor volume in early-stage Hodgkin lymphoma in the standard arm of the $\mathrm{H} 10$ trial. Blood 2018;131:1456-1463.

9. Goyal G, Tella SH, Funni S, et al. Association between facility volume and mortality of patients with classic Hodgkin lymphoma. Cancer 2020;126:757-764. 